\title{
Experimental Evaluation of a Low-Voltage Power Distribution Cable Model Based on a Finite-Element Approach
}

\author{
Georgios T. Andreou, Member, IEEE, and Dimitris P. Labridis, Senior Member, IEEE
}

\begin{abstract}
The authors presented in a former paper, a thorough study concerning the electrical parameters of low-voltage power distribution cables widely used in residential networks in the frequency range up to $100 \mathrm{MHz}$. The calculation of the electrical parameters was based on a finite-element procedure with respect to the cables' normal operational conditions. In this paper, a cable model is presented based on the above study. Approximations that are made are analyzed and validated. Moreover, an experimental setup is used to validate the cable model, which includes various and both single-path and multipath cable networks in order to achieve generality. Experimental results are analyzed, and theoretical difficulties based on the cables' nature are denoted.
\end{abstract}

Index Terms-Cable modeling, finite-element method (FEM), power-line communications.

\section{INTRODUCTION}

$\mathbf{P}$ OWER-LINE communications technology has improved rapidly over the last decade, resulting in complete and reliable systems, which can be used for communication over power distribution networks. This, however, is mainly the result of great improvements in encoding and multiplexing techniques. On the other hand, several attempts have been made concerning the proper modeling of low-voltage (LV) power distribution networks in consumer premises as communication media. Many of the models proposed in the literature attempt to describe the cables which comprise these networks by using their lumped or distributed electrical parameters (i.e., their resistance $R^{\prime}$, inductance $L^{\prime}$, conductance $G^{\prime}$, and capacitance $C^{\prime}$ per unit length [1]-[3]). Until recently, however, there was a lack of research concerning the theoretical estimation of these parameters.

In a former paper, the authors presented a thorough study concerning the exact estimation of the electrical parameters of LV power distribution cables widely used in residential networks, in the frequency range up to $100 \mathrm{MHz}$ [4]. A finite-element procedure was used for the calculations, with respect to the cables' normal operational conditions. The results were compared subsequently with corresponding values obtained by theoretical calculation methods.

In this paper, a cable model is presented based on the aforementioned results. In the first part of this paper, the model is described and the approximations made are explained and tested with respect to the cable's characteristic impedance $Z_{0}$

Manuscript received July 20, 2006. Paper no. TPWRD-00395-2006.

The authors are with the Department of Electrical and Computer Engineering, Aristotle University of Thessaloniki, Thessaloniki 54124, Greece (e-mail: gandreou@auth.gr; labridis@auth.gr).

Digital Object Identifier 10.1109/TPWRD.2007.900296

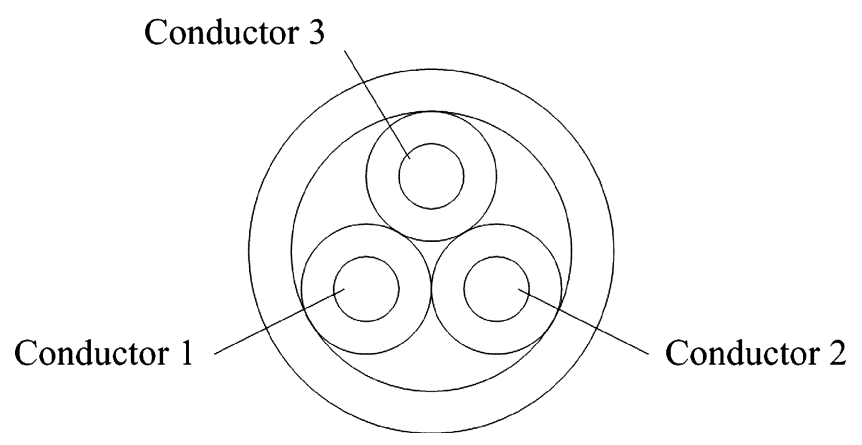

Fig. 1. Typical conductor arrangement for an NYM cable.

and propagation constant $\gamma$. In the second part, the experimental setup used for the validation of the model is presented. The various cases examined are demonstrated, and the experimental results are analyzed and compared to the values obtained by the theoretical model.

All results refer to a typical NYM $3 \times 2.5-\mathrm{mm}^{2} \mathrm{LV}$ cable (Fig. 1) without limiting the generality of the model. The examined frequency range reaches up to $30 \mathrm{MHz}$ since this is the typical frequency range utilized by the up-to-date power-line communications technology.

\section{THeORETICAL CABLE Model}

The cable model is based on properly estimated distributed electrical parameters. We will consider the cable to be lossless; therefore, throughout the calculations, we will only use its inductance per unit length $L^{\prime}$ and capacitance per unit length $C^{\prime}$. However, in this first part of the work we will also consider the cable's resistance per unit length $R^{\prime}$ and conductance per unit length $G^{\prime}$ so as to validate the above approximation. A more detailed study concerning the values used below can be found in [4].

\section{A. Resistance Per Unit Length}

The values used concerning the cable's resistance per unit length $R^{\prime}$ are calculated with the aforementioned finite-element procedure of [4]. The values are plotted versus the current frequency in Fig. 2.

\section{B. Inductance Per Unit Length}

Again, the values used concerning the cable's inductance per unit length $L^{\prime}$ are calculated with the aforementioned finite-element procedure of [4]. The values are plotted versus the current frequency in Fig. 3. 


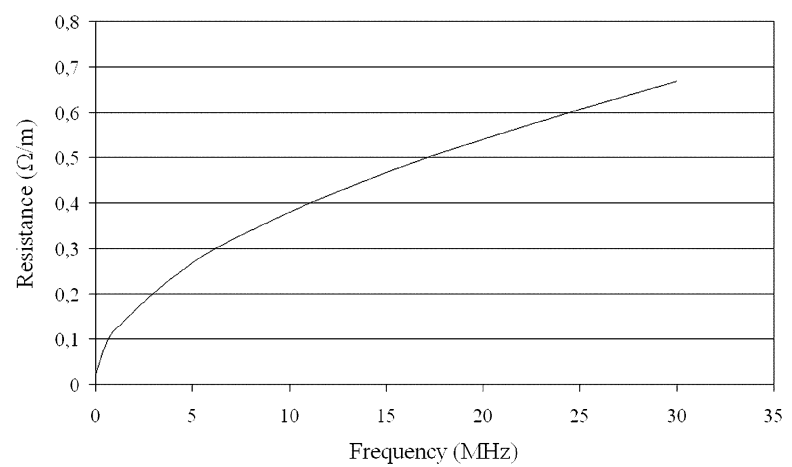

Fig. 2. Resistance per unit length for an NYM, $3 \times 2.5-\mathrm{mm}^{2}$ cable.

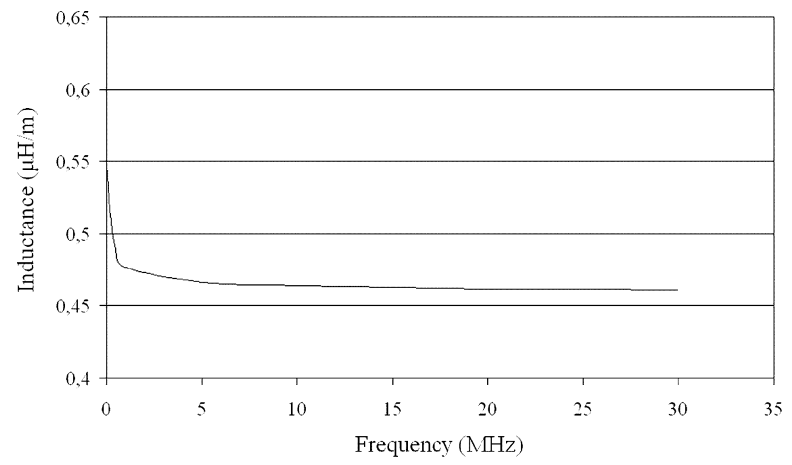

Fig. 3. Inductance per unit length for an NYM, $3 \times 2.5-\mathrm{mm}^{2}$ cable.

\section{Capacitance Per Unit Length}

As shown in [4], the capacitance per unit length $C$ of an LV power distribution cable presents us with the most theoretical problems. We can account for the partial capacitances between the conductors, but due to the absence of a metallic cover over the conductors, the analytical calculation of the corresponding partial capacitance between any of the conductors and earth is practically impossible, as in this case the extent of the electrostatic field is indeterminable [5]. The error factor, however, due to the absence of the partial capacitances to earth in the theoretical calculations is generally small because of the cable's small dimensions compared to its distance from earth, as we will see later.

The absence of a metallic cover over the conductors also makes the cable vulnerable to other adjacent electric-field sources, which can also not be accounted for. This will raise locally the cable's overall capacitance in a more or less random way, producing some substantial narrowband deviations in our results.

The cable's capacitance values used in the model presented here were calculated with the reciprocity theorem analyzed in [4]. The values for the permittivity of the dielectric separating the cable's conductors (PVC as in [6]) can be found in [7], and are plotted versus frequency in Fig. 4. The corresponding cable's capacitance-per-unit-length values are plotted versus frequency in Fig. 5.

\section{Conductance Per Unit Length}

The values for the cable's conductance per unit length $G^{\prime}$ are calculated theoretically as explained in [4], and they are plotted versus frequency in Fig. 6.

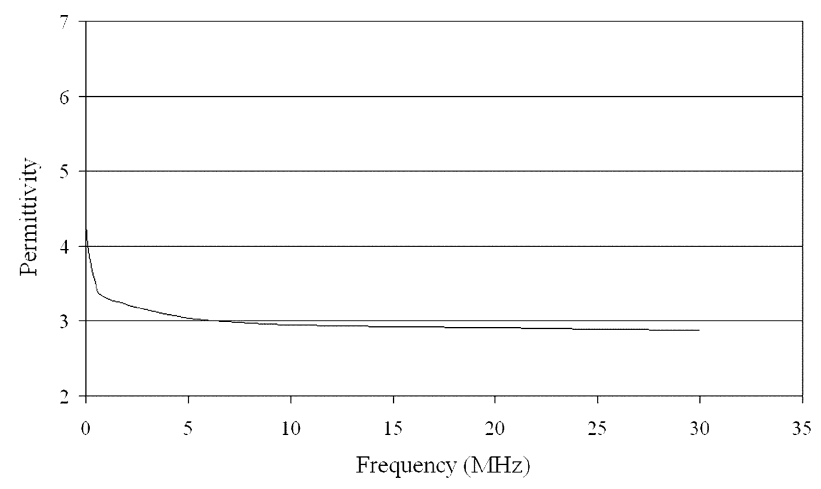

Fig. 4. Dielectric permittivity for an NYM, $3 \times 2.5-\mathrm{mm}^{2}$ cable.

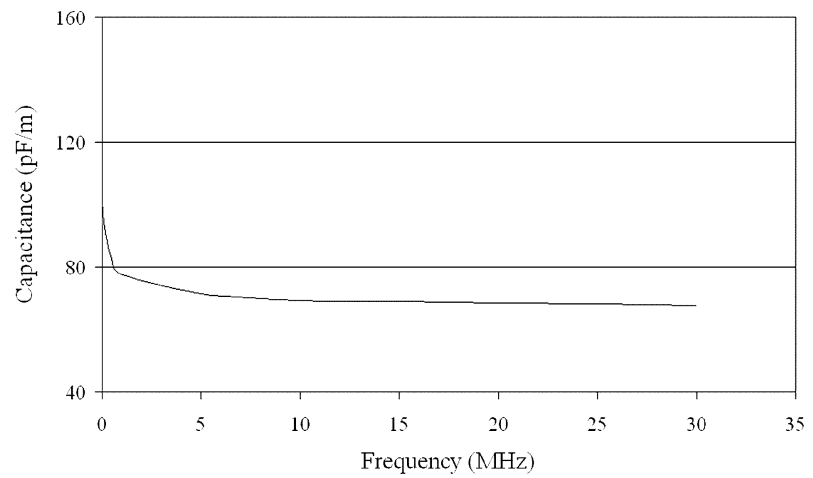

Fig. 5. Capacitance per unit length for an NYM, $3 \times 2.5-\mathrm{mm}^{2}$ cable.

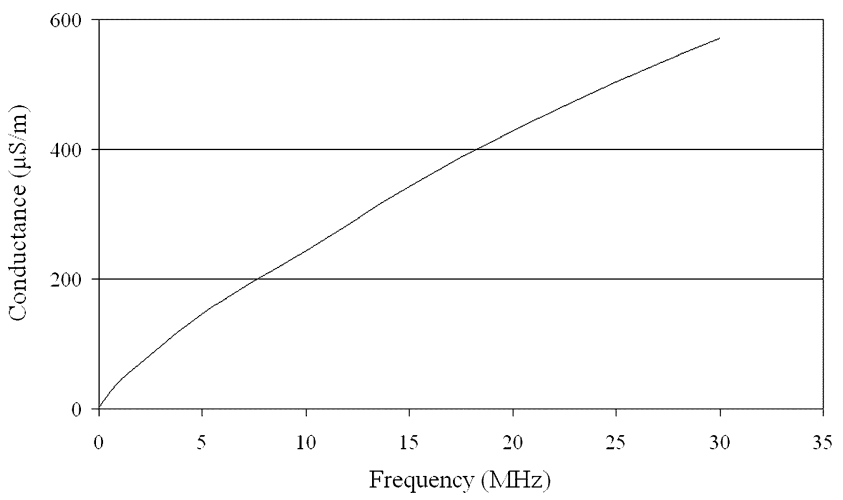

Fig. 6. Conductance per unit length for an NYM, $3 \times 2.5-\mathrm{mm}^{2}$ cable.

The corresponding values concerning the loss tangent of the cable's dielectric material can also be found in [7] and are plotted versus frequency in Fig. 7.

\section{E. Validation of Approximations}

Next, we will examine the variation in the cable's characteristic impedance $Z_{0}$ and propagation constant $\gamma$ because of the approximation made for the model presented here, regarding the cable as lossless.

The characteristic impedance of a uniform transmission line can be generally calculated with (1) [8] as follows:

$$
Z_{0}=\sqrt{\frac{R+j \omega L}{G+j \omega C}} .
$$




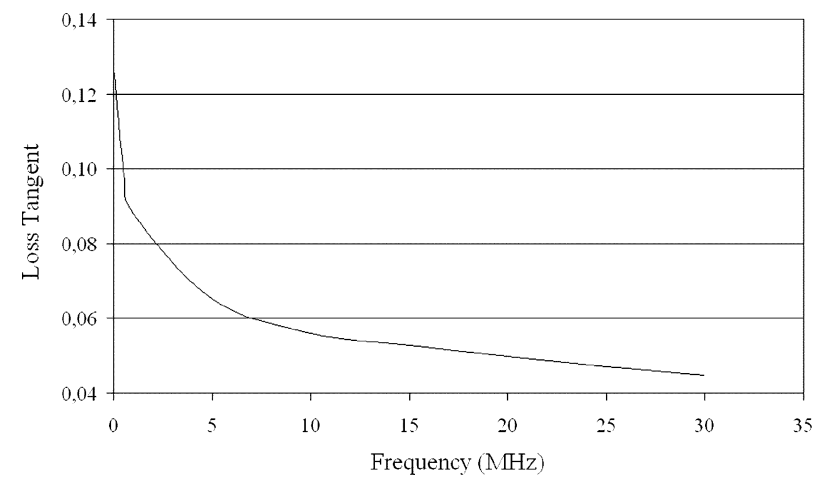

Fig. 7. Dielectric loss tangent for an NYM, $3 \times 2.5-\mathrm{mm}^{2}$ cable.

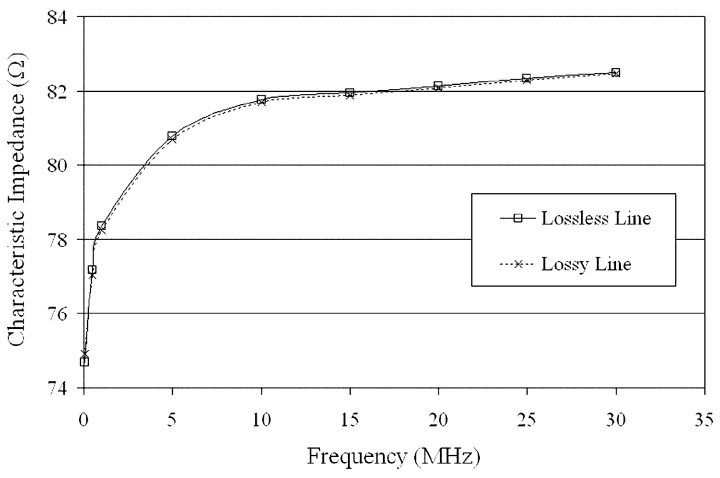

Fig. 8. Modulus of the characteristic impedance of an NYM, $3 \times 2.5-\mathrm{mm}^{2}$ cable when considered lossless and lossy, respectively.

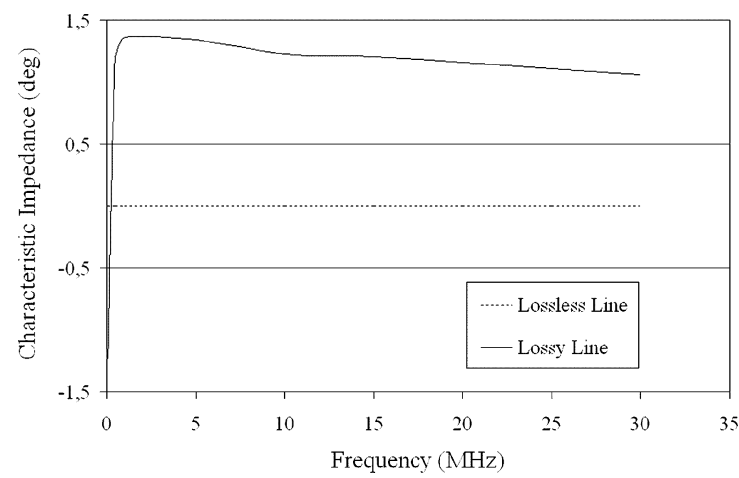

Fig. 9. Phase of the characteristic impedance of an NYM, $3 \times 2.5-\mathrm{mm}^{2}$ cable when considered lossless and lossy, respectively.

When $R$ and $G$ are small or when the frequency is large, so that $\omega L \gg R$ and $\omega C \gg G$, (1) reduces to [8]

$$
Z_{0}=\sqrt{\frac{L}{C}}
$$

Figs. 8 and 9 show the variation of the modulus and phase, respectively, of the cable's characteristic impedance, when the cable is considered as a lossy or lossless transmission line. The values for the respective cable's electrical parameters are taken as explained in the previous section of this paper.

For very low frequencies (up to about $10 \mathrm{kHz}$ ), the cable cannot be considered as a lossless transmission line, because the condition $\omega L \gg R$ does not hold. This frequency range is therefore omitted in the above figures, so as to improve clarity in the remaining frequency range.

As shown in Figs. 8 and 9, the cable can be very accurately considered as a lossless transmission line above the frequency of $10 \mathrm{kHz}$, as the deviation for the characteristic impedance's modulus remains below $0.33 \%$ for the whole remaining frequency range. The respective phase deviation remains for the same frequency range below $1.4^{\circ}$.

\section{F. Input Impedance of Lossless Transmission Line}

Matick shows in [8] that the impedance of a transmission line not terminated in its characteristic impedance, at any point $\mathrm{x}$ and looking toward the load, can be calculated with (3)

$$
Z_{x}=Z_{0} \frac{Z_{\ell}+Z_{0} \frac{e^{-\gamma x}-e^{-2 \gamma l} e^{\gamma x}}{e^{-\gamma x}+e^{-2 \gamma l} e^{\gamma x}}}{Z_{0}+Z_{\ell} \frac{e^{-\gamma x}-e^{-2 \gamma l} e^{\gamma x}}{e^{-\gamma x}+e^{-2 \gamma l} e^{\gamma x}}}
$$

where $Z_{0}$ is the characteristic impedance of the transmission line, $Z_{\ell}$ is its termination resistance, $\ell$ is its length, and $\gamma$ is its propagation constant.

If we let $x$ go to zero, determining the input impedance of the total line, (3) is then simplified to [8]

$$
Z_{\text {in }}=Z_{0} \frac{Z_{\ell}+Z_{0} \tanh (\gamma l)}{Z_{0}+Z_{\ell} \tanh (\gamma l)}
$$

For a lossless line, it will be [8]

$$
\gamma=j \beta=j \frac{2 \pi}{\lambda_{w}}
$$

where $\lambda_{w}$ is the wavelength of the applied frequency. Substituting (5) into (4) will yield

$$
Z_{\text {in }}=Z_{0} \frac{Z_{\ell}+j Z_{0} \tan \left(\frac{2 \pi \ell}{\lambda_{w}}\right)}{Z_{0}+j Z_{\ell} \tan \left(\frac{2 \pi \ell}{\lambda_{w}}\right)} .
$$

Equation (6) was used to validate the results obtained by the above theoretical analysis compared to the experimental results that follow.

\section{VALIDATION OF THEORETICAL MOdEL}

\section{A. Experimental Setup}

The experimental setup used to validate the theoretical analysis was built in regard to the normal operational conditions of the cable under study in a residential LV power distribution network. Therefore, a number of plastic cable ducts were fixed on an ordinary wall (without existing internal wiring), simulating the cable path of an ordinary residential power circuit. The duct arrangement appears in Fig. 10.

Within these ducts, a number of different cable configurations were used for the measurement procedure. During the experiments, an HP 8714ES network analyzer was used to measure the input impedance of the various circuits formed in the ducts by 


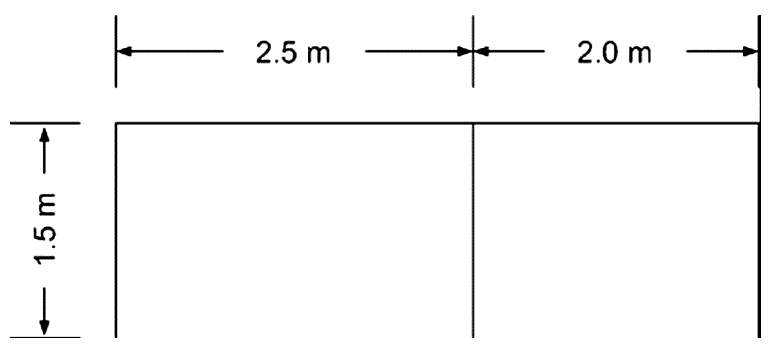

Fig. 10. Duct arrangement for experimental setup.

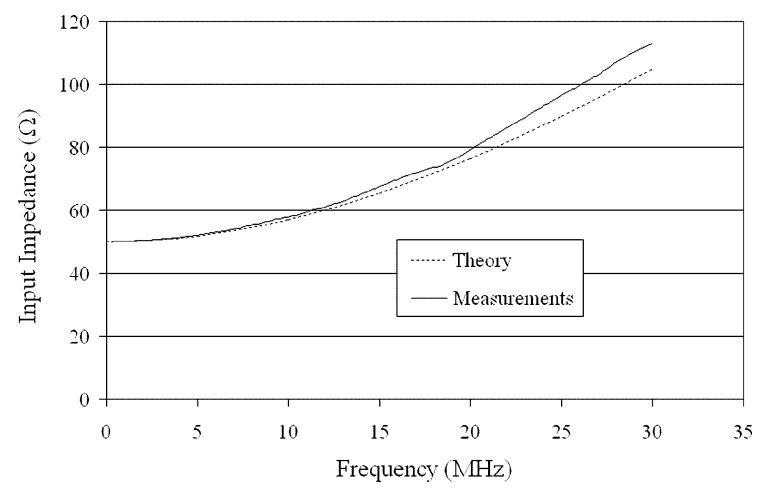

Fig. 11. Modulus of input impedance for Case 1: single NYM $3 \times 2.5-\mathrm{mm}^{2}$ cable, 1-m length.

the cables under study. The lower frequency limit of the measurements was set to $300 \mathrm{kHz}$, which was the lower limit of the network-analyzer frequency range. The measurements were conducted up to the frequency of $30 \mathrm{MHz}$, a typical power-line communications upper frequency limit.

The network analyzer was calibrated prior to any measurement set with the HP 85032E Type N calibration kit. Each calibration procedure included the configuration of the network analyzer with three standardized terminations implementing an open circuit, a short circuit, and a $50-\Omega$ termination resistance, respectively.

For every measurement, conductors 1 and 2 (Fig. 1) at a free cable end were attached to one port of the network analyzer, whereas conductor 3 was left open. The other cable ends were terminated with $50-\Omega$ termination resistances.

In the following paragraphs, various cases used during the measurement procedure are analyzed, and the results obtained are presented and compared to the respective theoretical calculations.

\section{B. Case A: Single Paths}

The first measurements regarding single cables were laid inside the ducts. The cable lengths used were 1,2, and $6 \mathrm{~m}$. The cable ends not attached to the network analyzer were terminated with a 50- $\Omega$ termination resistance. In Figs.11-16, experimental results are presented and compared to corresponding theoretical calculations for three cases (Case 1, Case 2, and Case 3), corresponding to the above three cable lengths $(1,2$, and $6 \mathrm{~m})$, respectively. Two figures correspond to every case, one showing the modulus of the cable's input impedance and one showing the respective phase.

In most cases, the above figures show an acceptable convergence between theoretical and experimental results. However,

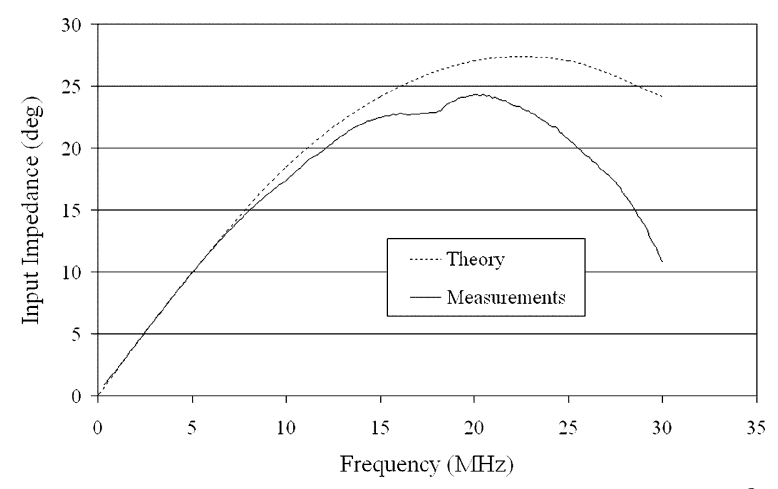

Fig. 12. Phase of input impedance for Case 1: single NYM $3 \times 2.5-\mathrm{mm}^{2}$ cable, 1-m length.

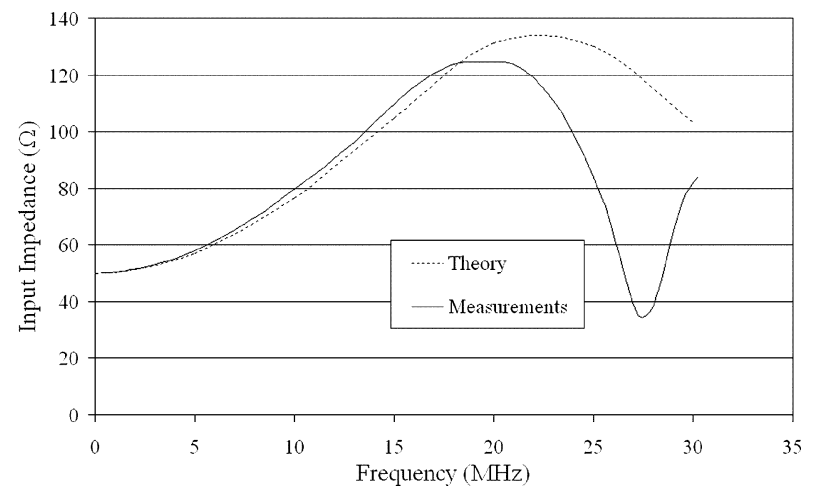

Fig. 13. Modulus of input impedance for Case 2: single NYM $3 \times 2.5-\mathrm{mm}^{2}$ cable, 2-m length.

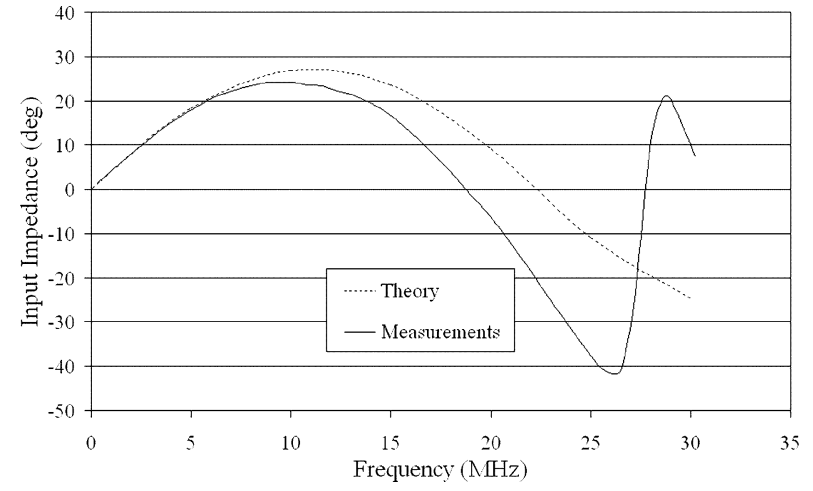

Fig. 14. Phase of input impedance for Case 2 : single NYM $3 \times 2.5-\mathrm{mm}^{2}$ cable, 2-m length.

there are small frequency ranges where the cable shows a significant capacitive behavior not anticipated by theory. The explanation lies in the aforementioned theoretical inadequacy to estimate accurately the cable's capacitance to earth and to the adjacent electric-field sources, which exist in any residential building. As an example, the greatest observed deviation in the above figures [about $80 \Omega$ or $70 \%$ in the modulus of the input impedance of a 2-m-long NYM, $3 \times 2.5-\mathrm{mm}^{2}$ cable at the frequency of $27.5 \mathrm{MHz}$ (Fig. 13) can be simulated with a respective increase in the cable's overall capacitance at the vicinity of that frequency, which can be caused by an equivalent outer capacitance within the order of $0.75 \mathrm{nF}$ at $27.5 \mathrm{MHz}]$.

Moreover, it can also be observed that the deviation of experimental results as compared to theoretical calculations decreases with the increase of the cable length, because of the respective 


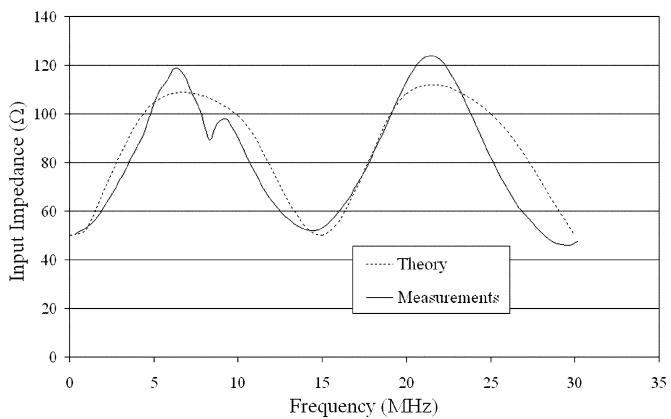

Fig. 15. Modulus of input impedance for Case 3: single NYM $3 \times 2.5-\mathrm{mm}^{2}$ cable, 6-m length.

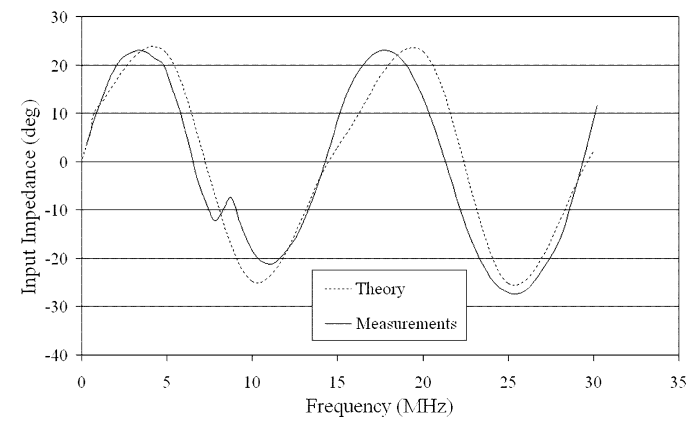

Fig. 16. Phase of input impedance for Case 3 : single NYM $3 \times 2.5-\mathrm{mm}^{2}$ cable, 6-m length.

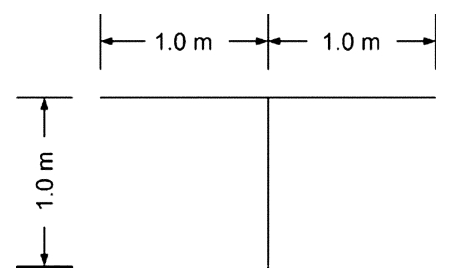

Fig. 17. Cable arrangement for Case 4.

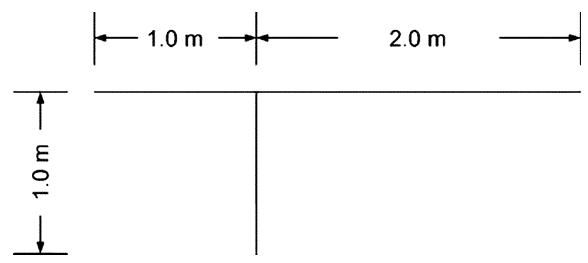

Fig. 18. Cable arrangement for Case 5 .

increase of the cable's inductance and capacitance, which results in the reduction of the influence of adjacent electric-field sources to the cable's impedance.

\section{Case B: Multipath Configurations}

Three different multipath cable configurations were used, arranged as shown in Figs. 17-19. The cable ends not attached to the network analyzer were also terminated with $50-\Omega$ termination resistance. Again, for every cable configuration, two figures exist, one showing the modulus of the cable's input impedance and one showing the respective phase.

Experimental results in Figs. 20-25 show again an acceptable convergence compared to theoretical calculations. Once more,

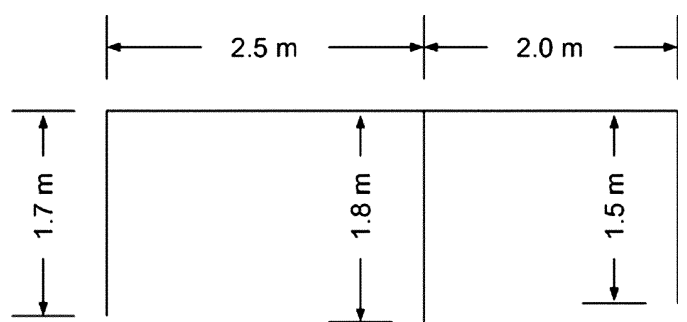

Fig. 19. Cable arrangement for Case 6.

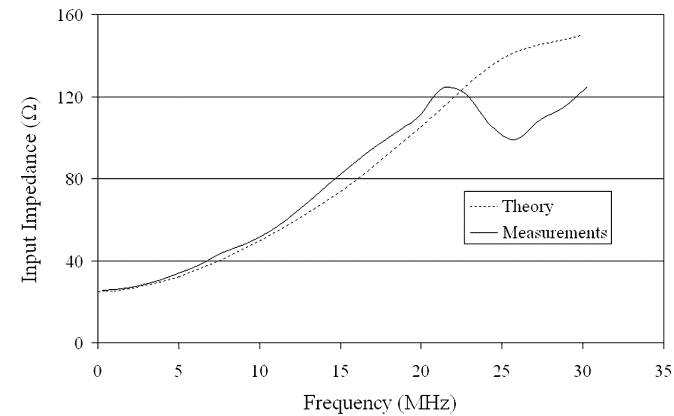

Fig. 20. Modulus of input impedance for Case 4.

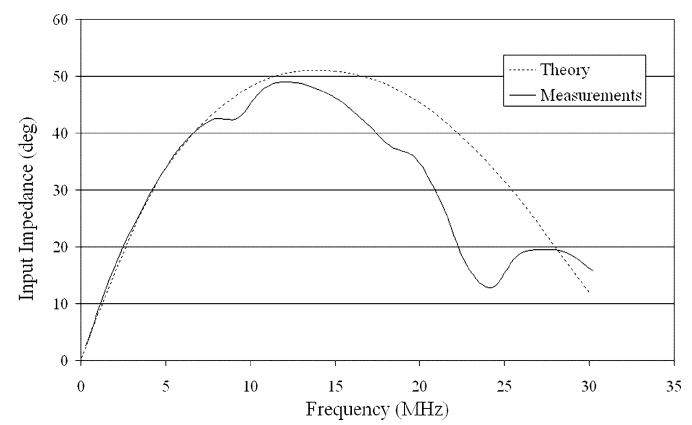

Fig. 21. Phase of input impedance for Case 4.

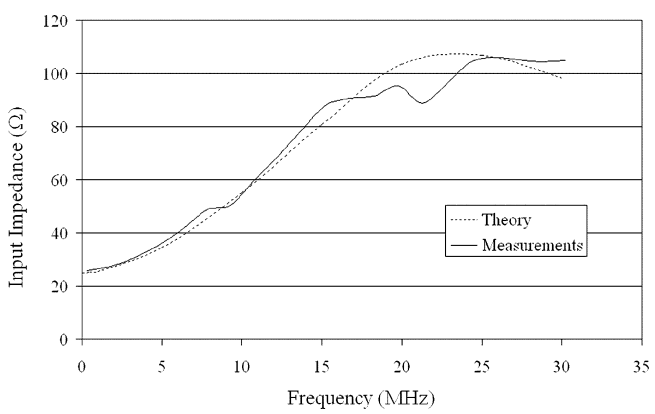

Fig. 22. Modulus of input impedance for Case 5 .

there are, however, small frequency ranges where the cable's capacitances to earth is and to adjacent electric-field sources, which cannot be estimated theoretically, causing significant deviations between theoretical and experimental results.

\section{CONCLUSION}

An experimental setup is used in this work to validate a cable model based on a theoretical analysis on the cables' electrical 


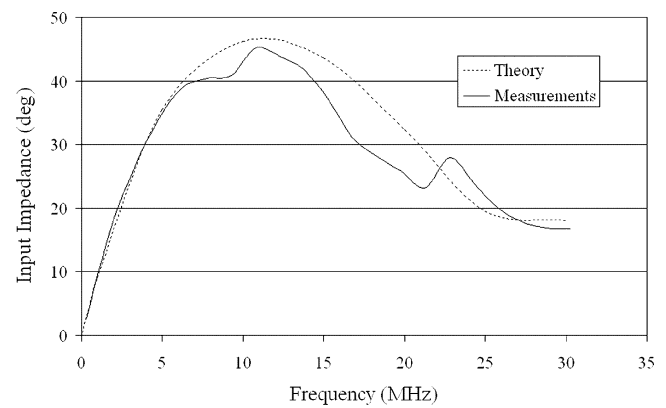

Fig. 23. Phase of input impedance for Case 5 .

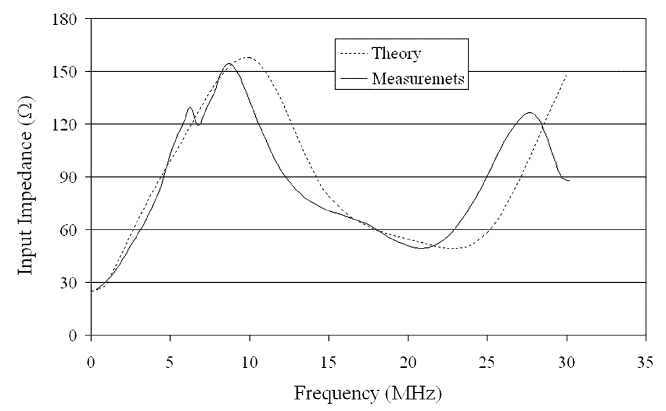

Fig. 24. Modulus of input impedance for Case 6.

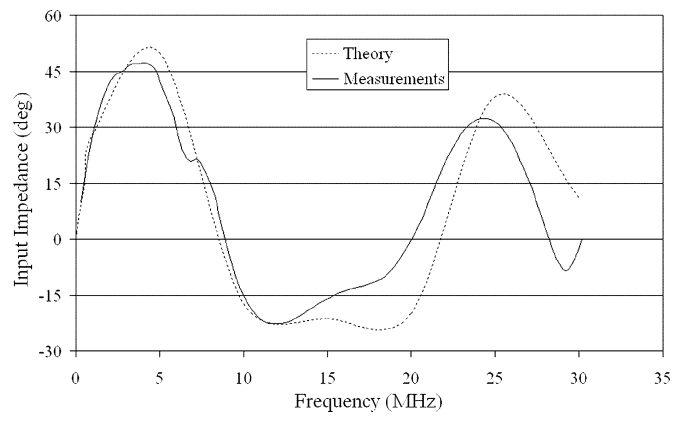

Fig. 25. Phase of input impedance for Case 6 .

parameters conducted by the authors in a former work. The frequency range used is that of a typical power-line communications system. At first, the values used for the electrical parameters of the cable type under study are presented. The approximations used in the model are analyzed and validated. Subsequently, the experimental setup is presented, and both simple and multipath cable configurations are used to validate the theoretical analysis.
Results show an acceptable convergence between theory and experiments concerning the various configurations' input impedance. There are, however, small frequency ranges with a substantial deviation due to the capacitive behavior of the cable not anticipated by theory. Nevertheless, this phenomenon was generally expected to appear because of the inherent theoretical inadequacy to accurately estimate the cable's capacitance to earth as well as to other adjacent electric-field sources.

\section{REFERENCES}

[1] D. Anastasiadou and T. Antonakopoulos, "An experimental setup for characterizing the residential power grid variable behavior," presented at the 6th Int. Symp. Power-Line Communications and Its Applications, Athens, Greece, 2002.

[2] I. C. Papaleonidopoulos, C. G. Karagiannopoulos, N. J. Theodorou, C. E. Anagnostopoulos, and I. E. Anagnostopoulos, "Modelling of indoor low voltage power-line cables in the high frequency range," presented at the 6th Int. Symp. Power-Line Communications and Its Applications, Athens, Greece, 2002.

[3] M. Zimmermann and K. Dostert, "A multipath model for the powerline channel," IEEE Trans. Commun., vol. 50, no. 4, pp. 553-559, Apr. 2002.

[4] G. T. Andreou and D. P. Labridis, "Electrical parameters of low-voltage power distribution cables used for power-line communications," IEEE Trans. Power Del., vol. 22, no. 2, pp. 879-886, Apr. 2007.

[5] L. Heinhold, Power Cables and their Application-Part 1. Berlin, Germany: Siemens Aktiengesellschaft, 1993, p. 331.

[6] R. Arora and W. Mosch, High Voltage Insulation Engineering. New Delhi, India: New Age International (P) Ltd., 2004, p. 242.

[7] A. von Hippel, Dielectric Materials and Applications. Norwood, MA: Artech House, 1994, pp. 329-330.

[8] R. E. Matick, Transmission Lines for Digital and Communication Networks. New York: IEEE Press, 1995, pp. 34-35.

Georgios T. Andreou (S'98-A'02-M'04) was born in Thessaloniki, Greece, on August 16, 1976. He received the Dipl.-Eng. degree from the Department of Electrical and Computer Engineering at the Aristotle University of Thessaloniki, Thessaloniki, in 2000.

Since 2001, he has been a postgraduate student in the Department of Electrical and Computer Engineering at the Aristotle University of Thessaloniki. His special interests are power system analysis and power-line communications.

Dimitris P. Labridis (S'88-M'90-SM'00) was born in Thessaloniki, Greece, on July 26, 1958. He received the Dipl.-Eng. and Ph.D. degrees from the Department of Electrical Engineering at the Aristotle University of Thessaloniki, Thessaloniki, in 1981 and 1989, respectively.

During 1982-2001, he was a Research Assistant and then a Lecturer and Assistant Professor at the Department of Electrical Engineering, Aristotle University of Thessaloniki. Since 2001, he has been Associate Professor at the same department. His special interests are power system analysis with special emphasis on the simulation of transmission and distribution systems, electromagnetic and thermal field analysis, numerical methods in engineering, artificial intelligence applications in power systems, and power-line communications. 